\title{
Compressively strained SiGe band-to-band tunneling model calibration based on p-i-n diodes and prospect of strained SiGe tunneling field-effect transistors
}

Kuo-Hsing Kao, Anne S. Verhulst, Rita Rooyackers, Bastien Douhard, Joris Delmotte, Hugo Bender, Olivier Richard, Wilfried Vandervorst, Eddy Simoen, Andriy Hikavyy, Roger Loo, Kai Arstila, Nadine Collaert, Aaron Thean, Marc M. Heyns, and Kristin De Meyer

Citation: Journal of Applied Physics 116, 214506 (2014);

View online: https://doi.org/10.1063/1.4903288

View Table of Contents: http://aip.scitation.org/toc/jap/116/21

Published by the American Institute of Physics

\section{Articles you may be interested in}

Tensile strained Ge tunnel field-effect transistors: $k \cdot p$ material modeling and numerical device simulation Journal of Applied Physics 115, 044505 (2014); 10.1063/1.4862806

Theory of Tunneling

Journal of Applied Physics 32, 83 (2004); 10.1063/1.1735965

Band structure, deformation potentials, and carrier mobility in strained $\mathrm{Si}, \mathrm{Ge}$, and $\mathrm{SiGe}$ alloys Journal of Applied Physics 80, 2234 (1998); 10.1063/1.363052

Band parameters for III-V compound semiconductors and their alloys Journal of Applied Physics 89, 5815 (2001); 10.1063/1.1368156

Boosting the on-current of a $n$-channel nanowire tunnel field-effect transistor by source material optimization Journal of Applied Physics 104, 064514 (2008); 10.1063/1.2981088

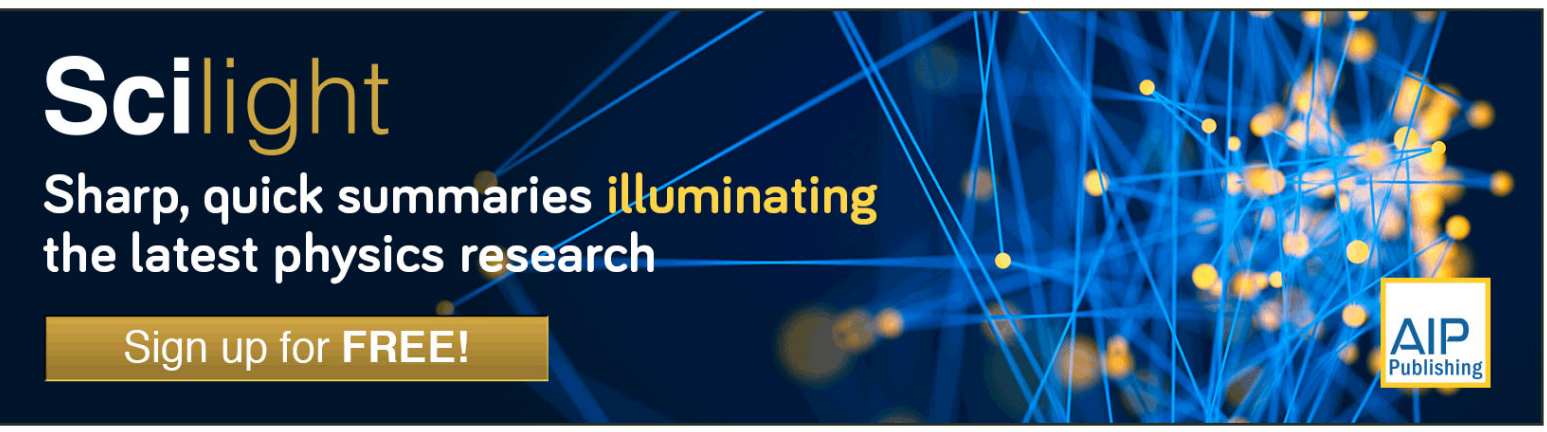




\title{
Compressively strained SiGe band-to-band tunneling model calibration based on p-i-n diodes and prospect of strained SiGe tunneling field-effect transistors
}

\author{
Kuo-Hsing Kao, ${ }^{1,2, a)}$ Anne S. Verhulst, ${ }^{1}$ Rita Rooyackers, ${ }^{1}$ Bastien Douhard, ${ }^{1}$ \\ Joris Delmotte, ${ }^{1}$ Hugo Bender, ${ }^{1}$ Olivier Richard, ${ }^{1}$ Wilfried Vandervorst, ${ }^{1}$ Eddy Simoen, ${ }^{1}$ \\ Andriy Hikavyy, ${ }^{1}$ Roger Loo, ${ }^{1}$ Kai Arstila, ${ }^{1}$ Nadine Collaert, ${ }^{1}$ Aaron Thean, ${ }^{1}$ Marc M. Heyns, ${ }^{1}$ \\ and Kristin De Meyer, ${ }^{1,2}$ \\ ${ }^{1}$ imec, Kapeldreef 75, 3001 Leuven, Belgium \\ ${ }^{2}$ Department of Electrical Engineering, KU Leuven, Leuven, Belgium
}

(Received 21 August 2014; accepted 21 November 2014; published online 3 December 2014)

\begin{abstract}
Band-to-band tunneling parameters of strained indirect bandgap materials are not well-known, hampering the reliability of performance predictions of tunneling devices based on these materials. The nonlocal band-to-band tunneling model for compressively strained SiGe is calibrated based on a comparison of strained SiGe p-i-n tunneling diode measurements and doping-profile-based diode simulations. Dopant and Ge profiles of the diodes are determined by secondary ion mass spectrometry and capacitance-voltage measurements. Theoretical parameters of the band-to-band tunneling model are calculated based on strain-dependent properties such as bandgap, phonon energy, deformation-potential-based electron-phonon coupling, and hole effective masses of strained SiGe. The latter is determined with a 6-band $k \cdot p$ model. The calibration indicates an underestimation of the theoretical electron-phonon coupling with nearly an order of magnitude. Prospects of compressively strained SiGe tunneling transistors are made by simulations with the calibrated model. (C) 2014 AIP Publishing LLC. [http://dx.doi.org/10.1063/1.4903288]
\end{abstract}

\section{INTRODUCTION}

SiGe band-to-band tunneling (BTBT) devices, such as tunneling diodes ${ }^{1-3}$ and tunneling field-effect transistors $(\text { TFETs })^{4-7}$ are of great interest to many researchers. The negative differential resistance (NDR) characteristic of tunneling diodes in forward bias is useful in oscillator and microwave amplifier applications. ${ }^{8}$ With their sub- $60 \mathrm{mV} / \mathrm{dec}$ subthreshold swing at room temperature, TFETs promise logic circuits with a reduced power consumption. Furthermore, the gateinduced drain leakage (GIDL) current of a conventional metal-oxide-semiconductor field-effect transistor (MOSFET) is also a BTBT current. This leakage current becomes more serious in strained SiGe MOSFETs due to the decreasing SiGe bandgap with strain. ${ }^{9}$ Therefore, well-calibrated parameters of the BTBT model for strained SiGe would be very useful.

The BTBT model of Kane ${ }^{10}$ has been successful in predicting BTBT currents of direct ${ }^{11-13}$ and indirect ${ }^{1-3,14-19}$ semiconductor devices since 1960. This well-known model still holds for tunneling events occurring in strained semiconductors. ${ }^{20}$ For indirect semiconductors, calibration of the electron-phonon interaction strength is still needed. In our study, the BTBT model for compressively strained SiGe is calibrated by comparing the experimental data and theoretical predictions of p-i-n diodes. A p-i-n diode allows accurate model calibration owing to the fact that the electrostatic potential profile from source to drain mainly depends on the doping concentration in the $\mathrm{p}$ and $\mathrm{n}$ regions. The gradients of

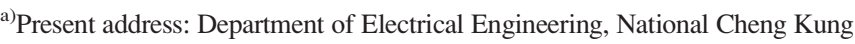
University, Tainan, Taiwan.
}

the doping profiles into the intrinsic region have limited impact on the BTBT current itself. Based on the calibrated BTBT model, an optimistic perspective of the compressively strained $\mathrm{SiGe}$ TFETs with various $\mathrm{Ge}$ concentrations is made. These predictions represent an upper bound of the device performance due to the favorable assumptions of the abrupt doping profiles and full strain.

We start in Sec. II with a discussion of the experiments including diode fabrication, secondary ion mass spectrometry (SIMS) analysis, and electrical characterization. In Sec. III, the essential models accounting for bandgap narrowing (BGN), Fermi level positioning, and BTBT for compressively strained SiGe are detailed. The BTBT model is calibrated in Sec. IV by comparing the theoretical results and experimental data. Then, a perspective of biaxially strained SiGe TFETs with compressive strain is presented in Sec. V.

\section{EXPERIMENT}

This section describes the diode fabrication, material analysis, and electrical characterizations including SIMS and current-voltage $(I V)$ measurements.

\section{A. Diode fabrication and material analysis}

Diodes are fabricated on boron-doped $(\mathrm{B}) \mathrm{p}^{+} \mathrm{Si}(001)$ blanket wafers with a resistivity of $0.005-0.010 \Omega \mathrm{cm}$. Epitaxial layers of heavily B-doped $\mathrm{p}^{+} \mathrm{Si}$, B-doped $\mathrm{p}^{+} \mathrm{SiGe}$, intrinsic SiGe (target thickness of $10 \mathrm{~nm}$ ), thin intrinsic $\mathrm{Si}$ capping (target thickness of $3 \mathrm{~nm}$, but actual thickness close to $10 \mathrm{~nm}$ ), and phosphorous-doped (P) $\mathrm{n}^{+} \mathrm{Si}$ (target thickness of $50 \mathrm{~nm}$ ) are grown subsequently using an ASM Epsilon ${ }^{\mathrm{TM}}$ 


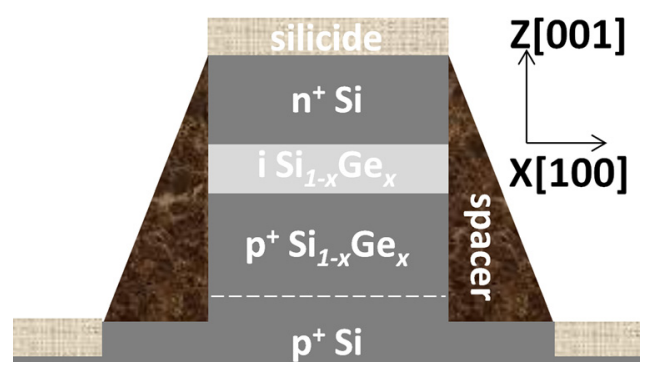

FIG. 1. Cross sectional view of a SiGe diode. The thin i-Si capping layer between the $\mathrm{i}-\mathrm{SiGe}$ and $\mathrm{n}^{+}-\mathrm{Si}$ is not shown.

3200 reactor $^{21}$ (Fig. 1). Starting with the B-doped SiGe layer, the highest temperature (about $550{ }^{\circ} \mathrm{C}$ ) is used for the epitaxial growth of the $\mathrm{P}$-doped $\mathrm{Si}$ layer. The diodes are defined by mesa patterning. After protecting the mesa sidewalls with a nitride spacer, contacts to the top and bottom junctions are formed by Ni-silicidation as shown in Fig. 1.

Two different diodes are used to perform the BTBT model calibration: strained $\mathrm{Si}_{1-x} \mathrm{Ge}_{x}$ diodes with $x=0.25$ (D1) and 0.45 (D2). The total strained SiGe layer thickness of each of the two diodes is targeted to be thinner than the critical layer thickness, such that relaxation with creation of dislocations can be averted. Therefore, the $\mathrm{p}-\mathrm{i}-\mathrm{n}$ diode is not fully $\mathrm{SiGe}$, but the $\mathrm{n}$-doped region is made of $\mathrm{Si}$. This choice has an added advantage that the Ni-alloying is limited to the doped $\mathrm{Si}$ region, which avoids spiking in the $\mathrm{SiGe}$ layer.
$100 \%$ strain and targeted Ge content in the $\mathrm{Si}_{1-x} \mathrm{Ge}_{x}$ layers of both diodes are confirmed by X-ray diffraction measurements (XRD not shown, examined before $n^{+}-\mathrm{Si}$ deposition) and Raman measurements (Table I). The nearly identical Raman peak frequency before and after $\mathrm{n}^{+}-\mathrm{Si}$ deposition combined with the XRD confirmation of strain before the $\mathrm{n}^{+}$-Si deposition confirms the strain preservation in the full diode stack.

Figs. 2(a) and 2(b) display the SIMS profiles for D1 and D2 used for BTBT model calibrations. To obtain the highest accuracy, separate ion bombardment species, $\mathrm{Cs}^{+}$(most sensitive for $\mathrm{P}$ ) and $\mathrm{O}_{2}{ }^{+}$(most sensitive for $\mathrm{B}$ ), are used on nearby samples on the same fabricated wafers. The ripples in the $\mathrm{P}$ signal are due to a selective growth process, which is based on a cyclic deposition and etch process, for the $\mathrm{n}^{+}-\mathrm{Si}$ deposition. $^{23,24}$ The same ripples are observed in the Ge-signal in the P-doped $\mathrm{Si}$ region (not shown in Fig. 2, Ge concentrations of $\max .0 .25 \%$, and therefore negligible impact on the Si properties).

The SIMS profiles shown in Fig. 2 have been modified compared to the raw data based on input from other characterization techniques. Modifications are: (i) the plateau value of the Ge profile obtained by SIMS has been converted to the Ge concentration confirmed by XRD and Raman and this conversion factor has been applied to the full Ge profile. (ii) The declining tails of the $\mathrm{B}$ and $\mathrm{P}$ profiles have been extrapolated from $3 \times 10^{18}$ down to $1 \times 10^{17} \mathrm{~cm}^{-3}$ (linear slope

TABLE I. Raman measurements of SiGe diodes D1 (targeted $x=0.25$ ) and D2 (targeted $x=0.45$ ) confirming the Ge concentration and strain as targeted. ${ }^{22}$

\begin{tabular}{|c|c|c|c|c|c|}
\hline Sample & $\begin{array}{l}\text { Si-Ge peak } \\
\quad\left[\mathrm{cm}^{-1}\right]\end{array}$ & $\begin{array}{l}\text { Si-Si peak } \\
{\left[\mathrm{cm}^{-1}\right]}\end{array}$ & $\begin{array}{c}\text { Measured Ge } \\
\text { content }(\%)\end{array}$ & $\begin{array}{c}\text { Strain }(\%) \\
\text { in SiGe layer based on } \\
\text { Si-Ge peak and targeted } \\
\text { SiGe content }\end{array}$ & $\begin{array}{l}\text { Strain }(\%) \text { in } \mathrm{SiGe} \text { layer } \\
\text { based on } \mathrm{Si}-\mathrm{Si} \text { peak } \\
\text { and targeted } \mathrm{SiGe} \text { content }\end{array}$ \\
\hline $\mathrm{D} 1 \mathrm{w} / \mathrm{o} \mathrm{n}^{+} \mathrm{Si}$ & 411.34 & 512.23 & 27 & -1.36 & -1.11 \\
\hline $\mathrm{D} 1 \mathrm{w} / \mathrm{n}^{+} \mathrm{Si}$ & 410.24 & 512.04 & 26 & -1.17 & -1.09 \\
\hline $\mathrm{D} 2 \mathrm{w} / \mathrm{o} \mathrm{n}^{+} \mathrm{Si}$ & 419.07 & 506.06 & 48 & -2.29 & -2.01 \\
\hline $\mathrm{D} 2 \mathrm{w} / \mathrm{n}^{+} \mathrm{Si}$ & 418.96 & 506.07 & 48 & -2.27 & -2.01 \\
\hline
\end{tabular}
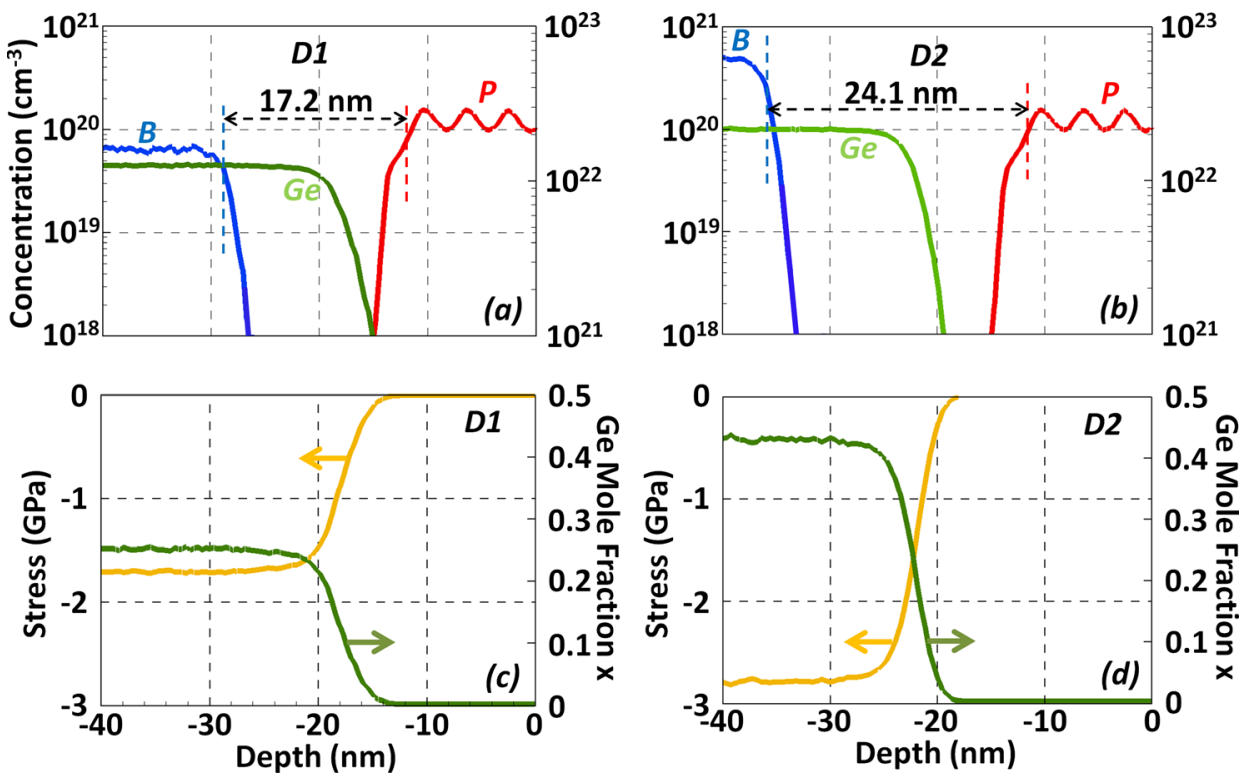

FIG. 2. Modified SIMS profiles of B, P (left axis) and Ge (right axis) of diodes (a) D1 and (b) D2. The dashed arrows indicate the intrinsic region thickness. Stress profiles orthogonal to the layer stack of (c) D1 and (d) D2 based on simulations with the modified central SIMS profiles. 
extension in the shown log-lin scale) and a background level at $1 \times 10^{17} \mathrm{~cm}^{-3}$ is taken, removing the noisy signal from background noise. Doping levels below $1 \times 10^{17} \mathrm{~cm}^{-3}$ no longer impact the electrostatic potential profile relevant to the BTBT generation rate. (iii) Modifications to the intrinsic region thickness are adopted based on transmission electron microscopy (TEM) input. The intrinsic region thickness (indicated by the dashed arrows in Fig. 2) is defined as the distance between the 2 points at which the concentration of the P- and B-profiles, respectively, has decreased to $50 \%$ of the plateau values. This intrinsic thickness is strongly linked to the electric field in the intrinsic region, which has an exponential impact on the BTBT rate and hence strongly impacts the BTBT calibration. TEM data (not shown), in particular, a high angle annular dark field scanning TEM (HAADF STEM) measurement display similar ripples as observed in the P- and Ge-SIMS-signal (Figs. 2(a) and 2(b)). The TEM ripples are identified to be the presence of $\mathrm{Ge}$ atoms in a $\mathrm{Si}$ matrix. Comparison of the HAADF STEM ripples with the Ge- and P-SIMS ripples hence allows to accurately extract the i-Si region thickness.

From the resulting best-estimate profiles of Figs. 2(a) and 2(b), we derive 3 representative profiles, with widest, central, and narrowest total i-region (i-Si and $\mathrm{i}-\mathrm{SiGe}$ ), respectively, based on representative error bars on TEM, scanning electron microscopy (SEM), and SIMS measurements. Error bars on both total i-region thickness (TEM, SEM, SIMS) and on the dopant profile steepness (SIMS only) are considered. The longest possible i-region thickness is combined with the steepest possible dopant profiles to create the widest profile and vice versa. Concerning dopant profile steepness, the raw Ge and B profile steepness determined by SIMS is considered as a lower limit given the roughness of the samples due to the presence of a silicide. The P-profile is steep since a non-silicided control wafer has been used. $100 \%$ dopant activation is assumed. The latter may not be correct for sample D2, which has a very high B-doping, but it has been verified through simulations that doping levels above $5 \times 10^{19} \mathrm{~cm}^{-3}$ are no longer affecting the electric field or electrostatic potential profile in the intrinsic region where the BTBT occurs, and hence the dopant activation above $5 \times 10^{19} \mathrm{~cm}^{-3}$ is not relevant to the BTBT calibration. The resulting central profiles for D1 and D2 are used for determining the stress profiles of Figs. 2(c) and 2(d), respectively, with SentaurusProcess (SProcess, see Sec. IV). ${ }^{25}$
To extract the most accurate profiles out of the set of widest, central, and narrowest, capacitance-voltage $(\mathrm{CV})$ measurements are used (see Sec. IV). It has been observed that any doping profiles which match a given $C V$ profile result in nearly the same $I V$ characteristics. The same correlation between $C V$ and $I V$ data has been observed in InGaAs calibration experiments. ${ }^{13}$ This correlation justifies possible overcorrections of the SIMS data (e.g., profile steepness).

\section{B. Diode electrical results}

IV measurements of diodes with different dimensions exhibit almost constant areal current as shown in Fig. 3. An obvious current spreading at high forward and reverse voltage is due to the series resistance. As will be discussed in Sec. IV, the diode with the smallest dimension will be used for calibration, such that the series resistance impact is the smallest. The constant areal current proves the opportunity to calibrate the BTBT model with simulations whereby only the center part of the diodes is considered. Any effect at the semiconductor/spacer interface (e.g., interfacial traps, dopant deactivation, and non-uniform strain profile) is not observable and can therefore be neglected.

Fig. 4 shows the $I V$ curves of the two diodes at different temperatures. Since BTBT current has less positive temperature dependence (relatively small activation energy) than the trap-assisted tunneling and Shockley-Read-Hall generation, ${ }^{26}$ BTBT is identified in Figs. 4(a) and 4(b) at $\mathrm{n}^{+}$bias $>0.8 \mathrm{~V}$ and $\mathrm{n}^{+}$bias $>1.7 \mathrm{~V}$, respectively.

\section{MODELS FOR COMPRESSIVELY STRAINED SiGe TUNNELING DEVICES}

The BTBT model calibration will be performed with the dynamic nonlocal BTBT model of SentaurusDevice (SDevice). ${ }^{25}$ To correctly calibrate the model for strained $\mathrm{SiGe}$, all contributing effects must be carefully incorporated, such as BGN caused by Ge-content, strain, or doping, effective mass modifications induced by Ge-content or strain and Fermi level $E_{F}$ positioning. Details of all the models employed in simulations will be discussed by considering a system of a biaxially compressively strained $\mathrm{SiGe}$ layer grown on a $\mathrm{Si}(001)$ substrate as shown in Fig. 5, and this configuration is representative for the central part of the diodes. The edge effect, such as the non-uniform strain profile at the mesa edges, is ignored since the current is
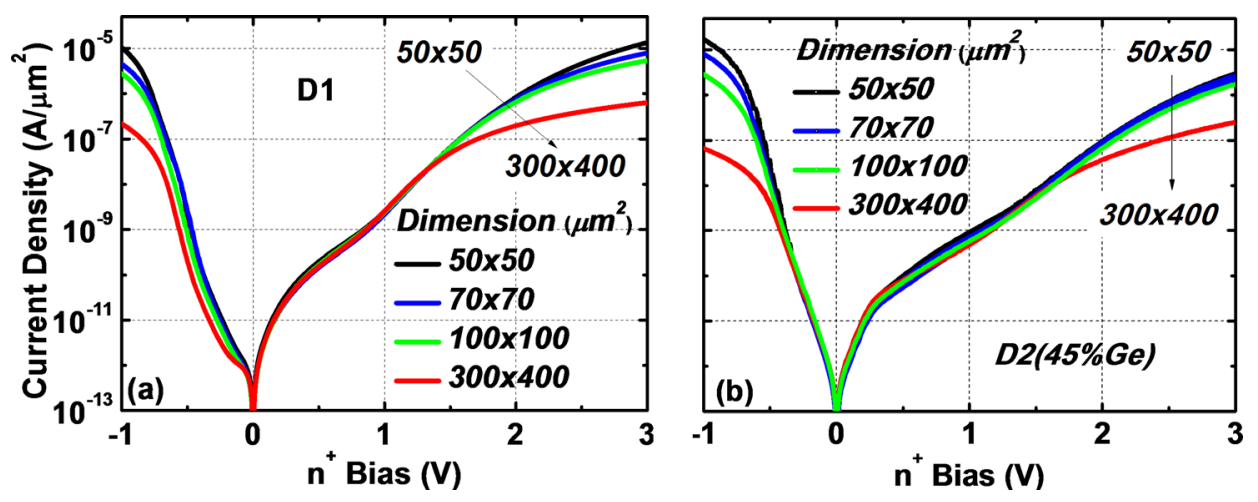

FIG. 3. Normalized current density of diodes (a) D1 and (b) D2 at room temperature. Even though D1 possesses a larger bandgap than D2, it shows higher reverse currents due to the thinner intrinsic layer (Fig. 2). 

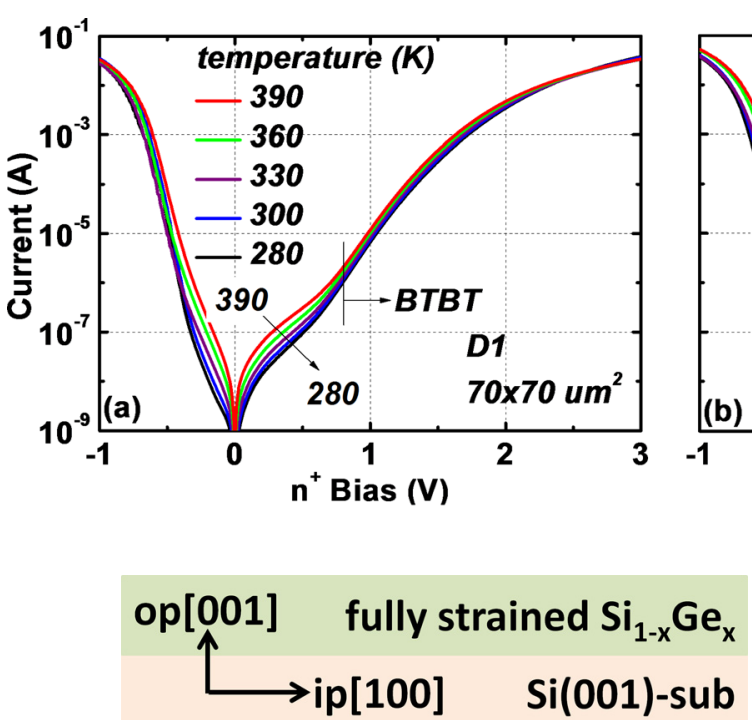

FIG. 5. A pseudomorphically grown $\mathrm{Si}_{1-x} \mathrm{Ge}_{x}$ layer on a $\mathrm{Si}(001)$ substrate. op[001] denotes the out-of-plane growth orientation being aligned to the tunneling direction [001] in the diode. ip[100] represents the in-plane direction.

completely dominated by the areal current as proven in Fig. 3. Note that the highest Ge content $x$ of strained $\mathrm{Si}_{1-x} \mathrm{Ge}_{x}$ will be limited to 0.7 owing to the critical thickness constraint caused by the lattice mismatch between $\mathrm{Si}$ and $\mathrm{Ge}$.

\section{A. Ge, strain, and doping induced bandgap narrowing}

Because the relaxed lattice constant of SiGe is larger than that of $\mathrm{Si}$, the pseudomorphically grown $\mathrm{SiGe}$ layer on a Si-sub is biaxially and compressively strained. Due to the Poisson effect, the SiGe layer is subject to an uniaxial tensile strain along the op[001]. The heavy hole $(\mathrm{HH})$ and light hole (LH) bands split off and the $\Delta_{6}$ conduction band valleys split into $\Delta_{4}$ and $\Delta_{2}$ because the lattice symmetry of the strained $\mathrm{SiGe}$ is broken. ${ }^{27} \mathrm{Fig}$. 6(a) presents the experimental bandgaps of relaxed ${ }^{28}$ and strained ${ }^{29} \mathrm{SiGe}$ as a function of Ge content $x$. While the conduction band offset $\Delta E_{c}$ between the $\mathrm{Si}$-sub and strained $\mathrm{SiGe}$ is almost zero in the range of $0 \leq x \leq 0.5$ (see Fig. 6(b)), the valence band offset $\Delta E_{v}$ varies linearly as a function of $x$. The latter has been proven experimentally $^{30}$ and the linear relation is given by $\Delta E_{v}$ $\approx 0.74 x \mathrm{eV}$ at room temperature as illustrated in Fig. 6(b). The impacts of Ge-content and strain on the bandgap and band alignment are considered in the SDevice simulations by the following approaches: (1) the relaxed SiGe bandgap is specified in the parameter file as a function of $x$. (2) The deformation potential model is activated resulting in the $V 1-\Delta_{4}$ bandgaps and $\Delta E_{v}$ as shown in Fig. 6.

Doping-dependent BGN is a result of five types of many-body interactions (electron-electron, hole-hole, electron-hole, electron-impurity, and hole-impurity). In the early 90s, Jain and Roulston developed an analytical formalism to calculate the various rigid shifts of the band edges for many semiconductors as a result of high doping, showing very good agreement with the experimental data. ${ }^{31}$ Since the $\mathrm{p}^{+}$and $\mathrm{n}^{+}$regions are heavily doped in our diodes, JainRoulston BGN model must be included in SDevice simulations. While the Jain-Roulston BGN parameters for $\mathrm{n}^{+} \mathrm{Si}$ have been well calibrated, those for $\mathrm{p}^{+}$strained $\mathrm{SiGe}$ can be empirically approximated by the ones for $\mathrm{p}^{+} \mathrm{Si}^{31}$

\section{B. Effective masses}

Both curvature masses and density of state (DOS) effective masses are needed to determine the parameters of the BTBT model in SDevice. The electron effective masses of the $\Delta_{4}$ valley of strained SiGe are nearly independent of strain and Ge content. ${ }^{32}$ To determine the hole effective masses, a 6-band $k \cdot p \operatorname{model}^{33}$ with a set of Luttinger parameters $^{27}$ is used to calculate the valence band structure of a relaxed $\mathrm{Si}_{1-x} \mathrm{Ge}_{x}$ layer as well as of a biaxially compressively strained $\mathrm{Si}_{1-x} \mathrm{Ge}_{x}$ layer grown on a $\mathrm{Si}(001)$ substrate as a function of Ge content $x$. As an example, Fig. 7(a) exhibits the electronic structure of the valence bands of strained $\mathrm{Si}_{0.75} \mathrm{Ge}_{0.25}$ in the in-plane and out-ofplane directions, while Fig. 7(b) projects two-dimensional

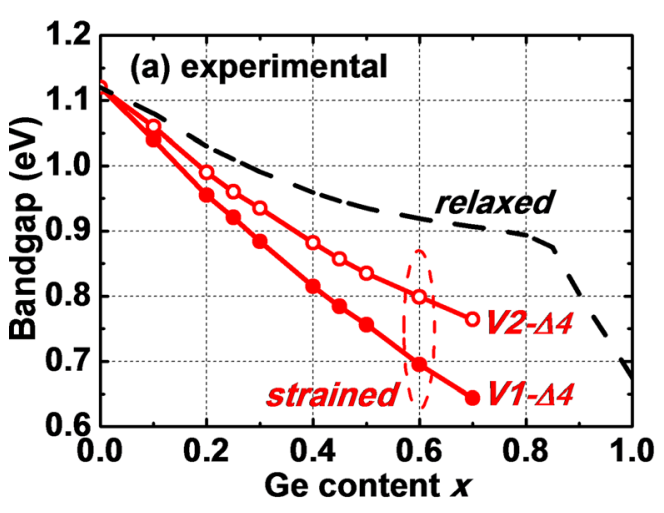

(b)

$E_{c}$

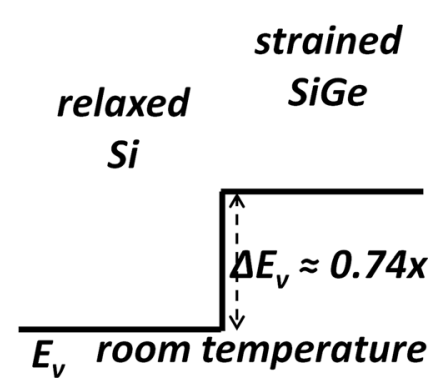

FIG. 6. Experimental bandgaps (a) and band alignment (b) of the strained $\mathrm{Si}_{1-x} \mathrm{Ge}_{x}$ pseudomorphically grown on $\mathrm{Si}(001)$-sub as a function of $x$ at room temperature. $V 1$ and $V 2$ represent the highest and the 2nd highest valence bands, respectively, as shown in Fig. 7(a). In (a), the dashed curve provides the relaxed $\mathrm{SiGe}$ bandgap. 

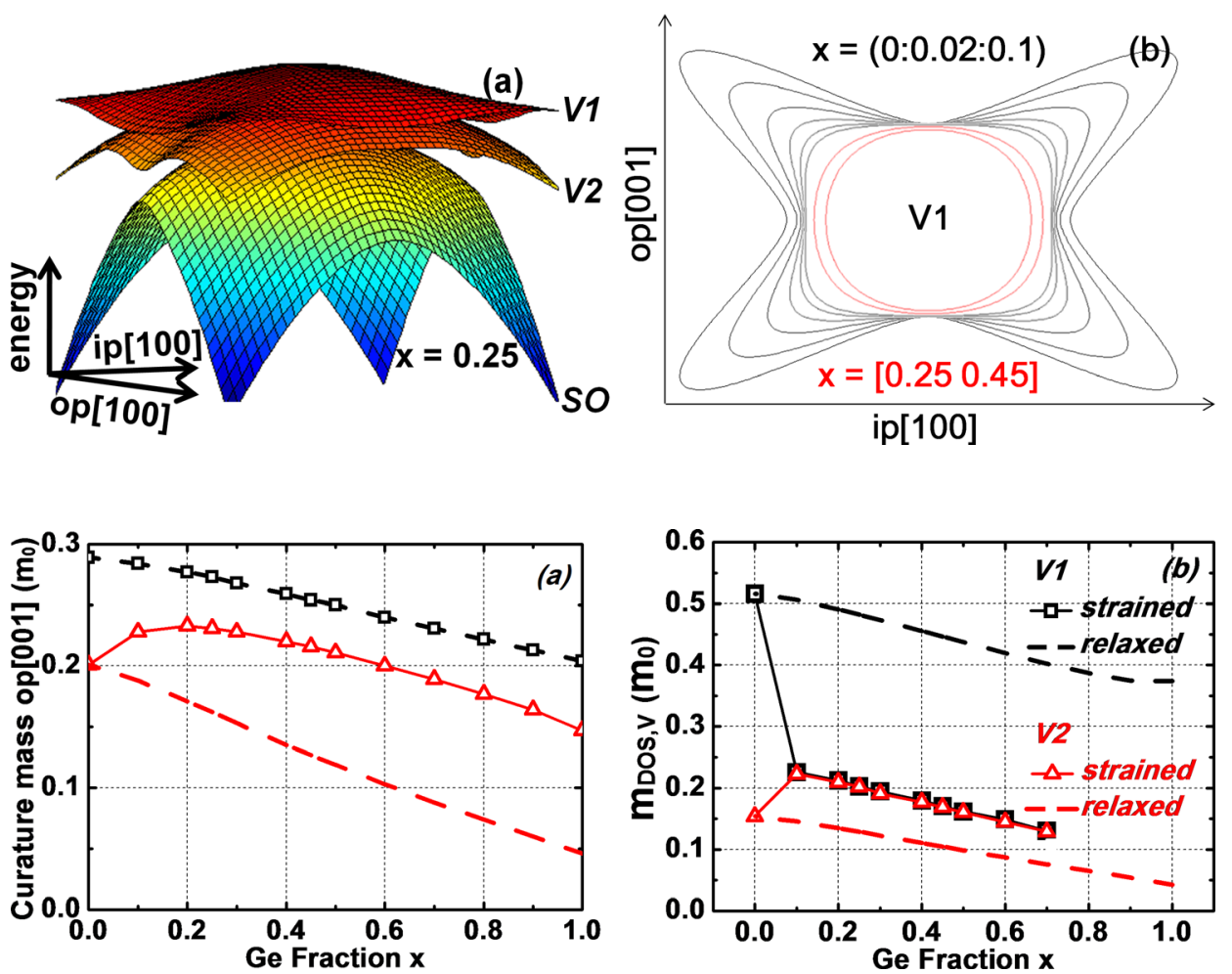

FIG. 7. (a) Valence band structure of a strained $\mathrm{Si}_{0.75} \mathrm{Ge}_{0.25}$ layer with notations of $V 1$ and $V 2$ for the highest and the 2nd highest valence bands, respectively. (b) The cross section of $V 1$ at $1 \mathrm{meV}$ below the top of the band for different $x$. The cross section area decreases monotonically with increasing $x$, with a very fast decrease for $0<x<0.1$. contours of $V 1$ for different Ge-content at $1 \mathrm{meV}$ below the band maximum.

From the band structure, the hole curvature and DOS $\left(m_{D O S, V}\right)$ effective masses of the relaxed and strained $\mathrm{Si}_{1-x} \mathrm{Ge}_{x}$ can be calculated and are shown in Fig. 8. The curvature masses, extracted at the band extremes, are in a good agreement with previous results. ${ }^{32,34}$ The $m_{D O S, V}$ in Fig. 8(b) is calculated from an integration of the available states within an energy window of $1 \mathrm{meV}$ from the top of the valence band in $\mathrm{k}$ space. A larger energy window for $m_{D O S, V}$ determination would lead to a larger $m_{D O S, V}$ due to the nonparabolic nature of the bands. ${ }^{32}$ At $x=0$ (relaxed $\mathrm{Si}$ ) in Fig. 8(b), $m_{D O S, V}$ is 0.516 and 0.154 for $V 1$ and $V 2$, respectively, corresponding to $\mathrm{HH}$ and $\mathrm{LH}$ bands. While the relaxed $m_{D O S, V}$ of the $V 1$ and $V 2$ band vary gradually with increasing Ge content, an abrupt decrease of $V 1$ and a kink in the curve of $V 2$ are observed for strained compositions with Ge-content between $x=0$ and 0.1 , which are consistent with previous results 35,36 as well. The rapid reduction in contour area observed in Fig. 7(b) can be directly linked to the abrupt drop of $V 1-m_{D O S, V}$ because the contour area (available states if only 2 directions are considered) is proportional to the $m_{D O S}$.

\section{Fermi level $E_{F}$ positioning}

By using the 6-band $k \cdot p$ method, the $E_{F}$ of a strained $\mathrm{p}^{+}$ $\mathrm{Si}_{1-x} \mathrm{Ge}_{x}(0 \leq x \leq 0.4)$ layer at $300 \mathrm{~K}$ as a function of doping concentration has been calculated ${ }^{36}$ as reproduced by the solid lines in Fig. 9. The $E_{F}$ predicted with SDevice by incorporating a multivalley band model (including $V 1$ and $V 2$ ) including non-parabolicity and activating the 6-band $k \cdot p$ DOS model for the valence band, agrees very well with this previous work as compared in Fig. 9. For instance, at
$1 \times 10^{20} \mathrm{~cm}^{-3}$ doping concentration and $x=0.4$, the SDevice $E_{F}$ is only $13 \mathrm{meV}(8 \%)$ smaller than the theoretically predicted value. This means that the electrostatic potential difference between the $\mathrm{p}^{+} \mathrm{SiGe}$ and $\mathrm{n}^{+} \mathrm{Si}$ of a $\mathrm{p}-\mathrm{i}-\mathrm{n}$ diode is smaller in SDevice resulting in a weaker electric field at zero bias. $I V$ curves predicted by SDevice, therefore, are expected to be shifted with at most $13 \mathrm{mV}$ towards higher reverse bias, and this is negligible in this study. Note that the n-type $\mathrm{Si}$ regions of the diodes are deposited together, and the doping concentration and $E_{F}$ are therefore independent of $\mathrm{SiGe}$ content.

\section{BTBT model for compressively strained $\mathrm{Si}_{1-x} \mathrm{Ge}_{\mathrm{x}}$}

Since the direct bandgap of the compressively strained $\mathrm{Si}_{1-\mathrm{x}} \mathrm{Ge}_{\mathrm{x}}$ is too large to contribute to the BTBT current when $x<0.7$, only the minimum $\Delta_{4}$ conduction band valleys are considered. The indirect BTBT generation rate $G$ of

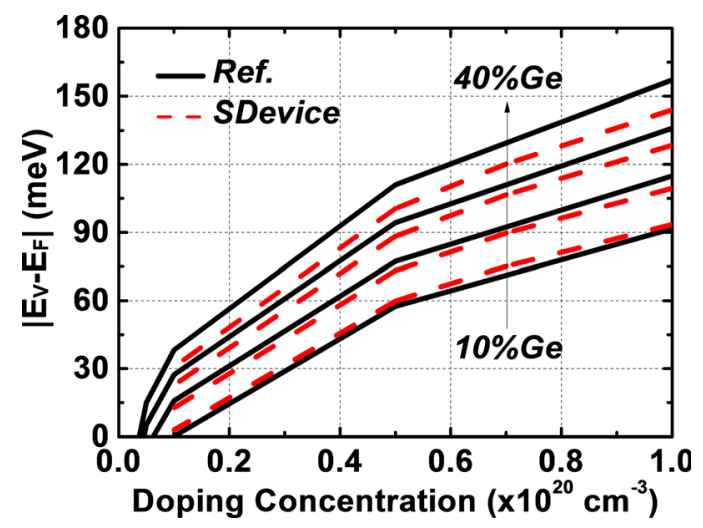

FIG. 9. Comparison of Fermi level energy of strained $\mathrm{Si}_{1-x} \mathrm{Ge}_{x}$ as a function of p-type doping concentration and different $x$ as extracted from Ref. 36 and as determined by SDevice. 
TABLE II. Physical parameters used in theoretical calculations of $A_{\text {ind }}$ and $B_{\text {ind }}$ for strained $\mathrm{Si}_{1-x} \mathrm{Ge}_{x}$ at various Ge mole fractions. $E_{g}^{r e}$ is the relaxed bandgap. All effective masses are in the units of the free electron mass $m_{o}$.

\begin{tabular}{|c|c|c|c|c|c|c|c|c|c|c|c|}
\hline$x$ & $\rho\left(\mathrm{kg} / \mathrm{m}^{3}\right)$ & $D_{T A}(\mathrm{eV} / \mathrm{m})$ & $\varepsilon_{T A}(\mathrm{meV})$ & $E_{g}^{r e}(\mathrm{eV})$ & $E_{g}^{V 1}(\mathrm{eV})$ & $E_{g}^{V 2}(\mathrm{eV})$ & $m_{L}$ & $m_{T}$ & $m_{D O S, C}$ & $m_{D O S, V 1}$ & $m_{D O S, V 2}$ \\
\hline 0 & 2329 & $1.69 \times 10^{10}$ & 19.0 & 1.12 & 1.12 & 1.12 & 0.92 & 0.19 & 0.32 & 0.52 & 0.15 \\
\hline 0.1 & 2673 & $1.64 \times 10^{10}$ & 18.0 & 1.08 & 1.040 & 1.060 & 0.92 & 0.19 & 0.32 & 0.23 & 0.22 \\
\hline 0.2 & 3008 & $1.60 \times 10^{10}$ & 16.9 & 1.03 & 0.955 & 0.990 & 0.92 & 0.19 & 0.32 & 0.21 & 0.21 \\
\hline 0.25 & 3171 & $1.58 \times 10^{10}$ & 16.4 & 1.01 & 0.921 & 0.960 & 0.92 & 0.19 & 0.32 & 0.20 & 0.20 \\
\hline 0.3 & 3332 & $1.55 \times 10^{10}$ & 15.9 & 0.991 & 0.884 & 0.935 & 0.92 & 0.19 & 0.32 & 0.20 & 0.19 \\
\hline 0.4 & 3646 & $1.51 \times 10^{10}$ & 14.8 & 0.959 & 0.815 & 0.882 & 0.92 & 0.19 & 0.32 & 0.18 & 0.18 \\
\hline 0.45 & 3800 & $1.48 \times 10^{10}$ & 14.3 & 0.946 & 0.784 & 0.857 & 0.92 & 0.19 & 0.32 & 0.17 & 0.17 \\
\hline 0.5 & 3951 & $1.46 \times 10^{10}$ & 13.8 & 0.935 & 0.756 & 0.835 & 0.92 & 0.19 & 0.32 & 0.16 & 0.16 \\
\hline 0.6 & 4245 & $1.42 \times 10^{10}$ & 12.8 & 0.919 & 0.695 & 0.799 & 0.92 & 0.19 & 0.32 & 0.15 & 0.15 \\
\hline \multirow[t]{2}{*}{0.7} & 4530 & $1.37 \times 10^{10}$ & 11.7 & 0.907 & 0.644 & 0.764 & 0.92 & 0.19 & 0.32 & 0.13 & 0.13 \\
\hline & Ref. 40 & 2737 & 38 & 28 & 29 & 29 & 32 & 32 & & This work & \\
\hline
\end{tabular}

Kane's model under an uniform electric field can be expressed by ${ }^{10,25}$

$$
\begin{gathered}
G=A_{\text {ind }}\left(\frac{F}{F_{0}}\right)^{2.5} \exp \left(-\frac{B_{\text {ind }}}{F}\right), \\
A_{\text {ind }}=\frac{g\left(m_{D O S, C} m_{D O S, V}\right)^{3 / 2}\left(1+2 N_{T A}\right) D_{T A}^{2}\left(q F_{0}\right)^{5 / 2}}{2^{21 / 4} h^{5 / 2} m_{r}^{5 / 4} \rho \varepsilon_{T A} E_{g}^{7 / 4}}, \\
B_{\text {ind }}=\frac{2^{7 / 2} \pi m_{r}^{1 / 2} E_{g}^{3 / 2}}{3 q h},
\end{gathered}
$$

where $F_{0}=1 \mathrm{MV} / \mathrm{cm}, F$ is the electric field, $h$ is Plank's constant, $\rho$ is the mass density, $q$ is the elementary charge, $g$ is equal to $2 \times 1 \times 4$, sequentially corresponding to the degeneracy of spin, of $V 1$ (or $V 2$ ) valence and of $\Delta_{4}$ conduction. The conduction band DOS mass $m_{D O S, C}$ of the $\Delta_{4}$ valleys is defined as the geometric mean of longitudinal $m_{L}$ and transverse $m_{T}$ effective masses ( $m_{L}$ and $m_{T}$ of strained SiGe are nearly independent of strain and Ge-content ${ }^{32}$ ), and $m_{D O S, V}$ is shown in Fig. 8(b). The reduced tunneling mass $m_{r}$ is determined by the curvature masses of conduction bands $\left(m_{e}\right)$ and valence bands $\left(m_{V 1}\right.$ and $\left.m_{V 2}\right)$ along the given tunneling direction, which is op[001] in this study. The curvature masses of $V 1$ and $V 2$ are shown in Fig. 8(a). Since the energy separation between $V 1$ and $V 2$ bands is only about $80 \mathrm{meV}$ at $x=0.5$, two BTBT paths are considered, namely, transitions $V 1-\Delta_{4}$ and $V 2-\Delta_{4}$. The indirect bandgaps $E_{g}$ presented in Fig. 6(a) are used. The transverse acoustic (TA) phonon deformation potential $D_{T A}$ and phonon energy $\varepsilon_{T A}$ of $\mathrm{SiGe}$ can be determined according to the previous studies. ${ }^{27,37,38}$ Note that we only take the TA phonons into account because they have the smallest phonon energy, the highest phonon occupation number and hence they can be expected to provide the main contribution to the indirect BTBT, even though experiments suggested that the TO phonon may also contribute to BTBT in silicon. ${ }^{39}$ We have not included TO phonon contributions at this point in the BTBT model, since there is no formula available for the corresponding TO deformation potential. Our calibration will indicate that the total electron-phonon coupling has been underestimated and hence our calibration will provide a value for the TO deformation potential. Table II lists all material and BTBT parameters of compressively strained SiGe.

$A_{\text {ind }}$ and $B_{\text {ind }}$ of strained $\mathrm{Si}_{1-x} \mathrm{Ge}_{x}$ grown on $\mathrm{Si}(001)$-sub from Table III are plotted against $x$ in Fig. 10. By comparing them with Figs. 6(a) and 8(b), one can find that the trends of $A_{\text {ind }}$ and $B_{\text {ind }}$ are dominated by the variations of the hole

TABLE III. Degeneracy factor $g$, electron curvature effective mass $m_{e}$, hole effective curvature mass $m_{V 1} / m_{V 2}$, and the reduced tunneling mass $m_{r}$ used in theoretical calculations of $A_{\text {ind }}$ and $B_{\text {ind }}$ for the indirect BTBT along op[001] in the strained $\mathrm{Si}_{1-x} \mathrm{Ge}_{x}$. All effective masses are in units of the free electron mass $m_{o}$. $A_{\text {ind }}$ and $B_{\text {ind }}$ are in units of $\mathrm{cm}^{-3} \mathrm{~s}^{-1}$ and $\mathrm{MV} \mathrm{cm}^{-1}$, respectively. Note that $g, m_{e}$, and $m_{V 1}$ (see Fig. 8(a), $V 1 \mathrm{corresponding}$ to $\mathrm{HH}$ in relaxed SiGe) are strainindependent. The calibrated values of $A_{\text {ind }}$ and $B_{\text {ind }}$ for strained $\mathrm{Si}_{1-x} \mathrm{Ge}_{x}$ at various $\mathrm{Ge}$ mole fractions are the presented values rescaled by factors 7.6 and $1 / 1.1$

\begin{tabular}{|c|c|c|c|c|c|c|c|c|c|c|}
\hline$x$ & $g$ & $m_{e}$ & $m_{V 1}$ & $m_{V 2}$ & $m_{r}^{V 1}$ & $m_{r}^{V 2}$ & $A_{\text {ind }}^{V 1}$ & $B_{i n d}^{V 1}$ & $A_{\text {ind }}^{V 2}$ & $B_{\text {ind }}^{V 2}$ \\
\hline 0 & 8 & $m_{T}$ & 0.289 & 0.201 & 0.115 & 0.098 & $0.976 \times 10^{15}$ & 27.4 & $0.194 \times 10^{15}$ & 25.3 \\
\hline 0.1 & 8 & $m_{T}$ & 0.284 & 0.228 & 0.114 & 0.104 & $0.296 \times 10^{15}$ & 24.4 & $0.317 \times 10^{15}$ & 24.0 \\
\hline 0.2 & 8 & $m_{T}$ & 0.277 & 0.233 & 0.113 & 0.105 & $0.301 \times 10^{15}$ & 21.4 & $0.304 \times 10^{15}$ & 21.8 \\
\hline 0.25 & 8 & $m_{T}$ & 0.273 & 0.231 & 0.112 & 0.104 & $0.294 \times 10^{15}$ & 20.2 & $0.299 \times 10^{15}$ & 20.7 \\
\hline 0.3 & 8 & $m_{T}$ & 0.268 & 0.228 & 0.111 & 0.104 & $0.295 \times 10^{15}$ & 18.9 & $0.284 \times 10^{15}$ & 19.9 \\
\hline 0.4 & 8 & $m_{T}$ & 0.259 & 0.220 & 0.110 & 0.102 & $0.304 \times 10^{15}$ & 16.6 & $0.284 \times 10^{15}$ & 18.1 \\
\hline 0.45 & 8 & $m_{T}$ & 0.254 & 0.216 & 0.109 & 0.101 & $0.303 \times 10^{15}$ & 15.6 & $0.279 \times 10^{15}$ & 17.2 \\
\hline 0.5 & 8 & $m_{T}$ & 0.250 & 0.211 & 0.108 & 0.100 & $0.300 \times 10^{15}$ & 14.7 & $0.274 \times 10^{15}$ & 16.5 \\
\hline 0.6 & 8 & $m_{T}$ & 0.240 & 0.200 & 0.106 & 0.097 & $0.318 \times 10^{15}$ & 12.9 & $0.267 \times 10^{15}$ & 15.2 \\
\hline 0.7 & 8 & $m_{T}$ & 0.231 & 0.189 & 0.104 & 0.095 & $0.318 \times 10^{15}$ & 11.4 & $0.261 \times 10^{15}$ & 14.0 \\
\hline
\end{tabular}
for $A_{i n d}$ and $B_{i n d}$, respectively.

Indirect BTBT [001] 

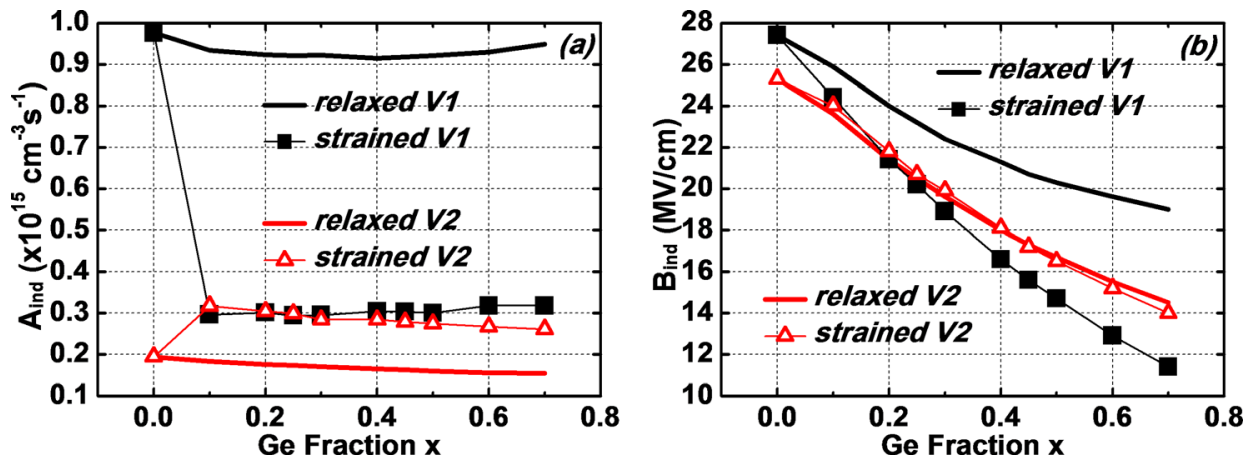

FIG. 10. Theoretical (a) $A_{\text {ind }}$ and (b) $B_{\text {ind }}$ for the indirect BTBT along op[001] in the $\mathrm{Si}_{1-x} \mathrm{Ge}_{x}$ layer grown on $\mathrm{Si}(001)$. All data can be found in Tables II, III, and IV.

TABLE IV. Same parameters as Table II but for tunneling along [001] in relaxed SiGe. $m_{L H}$ is the curvature mass of the LH band. Other required parameters of Eqs. (2) and (3) not tabulated here can be found in Tables II and III.

\begin{tabular}{|c|c|c|c|c|c|c|c|c|c|}
\hline$X$ & $m_{L H}$ & $m_{r}^{L H}$ & $m_{r}^{H H}$ & $m_{D O S, H H}$ & $m_{D O S, L H}$ & $A_{\text {ind }}^{L H}$ & $B_{\text {ind }}^{L H}$ & $A_{\text {ind }}^{H H}$ & $B_{\text {ind }}^{H H}$ \\
\hline 0 & 0.201 & 0.098 & 0.115 & 0.516 & 0.154 & $0.194 \times 10^{15}$ & 25.3 & $0.976 \times 10^{15}$ & 27.4 \\
\hline 0.1 & 0.188 & 0.095 & 0.114 & 0.506 & 0.146 & $0.183 \times 10^{15}$ & 23.6 & $0.933 \times 10^{15}$ & 25.9 \\
\hline 0.2 & 0.171 & 0.090 & 0.113 & 0.491 & 0.135 & $0.176 \times 10^{15}$ & 21.4 & $0.923 \times 10^{15}$ & 24.0 \\
\hline 0.25 & 0.162 & 0.087 & 0.112 & 0.483 & 0.129 & $0.173 \times 10^{15}$ & 20.5 & $0.921 \times 10^{15}$ & 23.2 \\
\hline 0.3 & 0.153 & 0.085 & 0.111 & 0.474 & 0.123 & $0.170 \times 10^{15}$ & 19.6 & $0.922 \times 10^{15}$ & 22.4 \\
\hline 0.4 & 0.135 & 0.079 & 0.110 & 0.456 & 0.110 & $0.165 \times 10^{15}$ & 18.0 & $0.915 \times 10^{15}$ & 21.3 \\
\hline 0.45 & 0.127 & 0.076 & 0.109 & 0.447 & 0.104 & $0.163 \times 10^{15}$ & 17.3 & $0.918 \times 10^{15}$ & 20.7 \\
\hline 0.5 & 0.119 & 0.073 & 0.108 & 0.438 & 0.098 & $0.160 \times 10^{15}$ & 16.7 & $0.921 \times 10^{15}$ & 20.3 \\
\hline 0.6 & 0.103 & 0.067 & 0.106 & 0.420 & 0.087 & $0.156 \times 10^{15}$ & 15.5 & $0.929 \times 10^{15}$ & 19.6 \\
\hline 0.7 & 0.088 & 0.060 & 0.104 & 0.403 & 0.076 & $0.154 \times 10^{15}$ & 14.5 & $0.949 \times 10^{15}$ & 19.0 \\
\hline
\end{tabular}

DOS mass and bandgap, respectively. Comparison is made with $A_{\text {ind }}$ and $B_{\text {ind }}$ of relaxed $\mathrm{Si}_{1-x} \mathrm{Ge}_{x}$ from Table IV. Table IV is an updated version of the parameters for relaxed $\mathrm{SiGe}$ in Ref. 41. Note that instead of collecting data from different literatures, ${ }^{41}$ in this study $D_{T A}$, electron/hole curvature, and DOS effective masses are determined with consistent deformation potentials and Luttinger parameters to what has been employed in SDevice as aforementioned for strained SiGe.

Fig. 11 shows the total theoretical BTBT generation rate including the transitions of $\Delta_{4}-V 1$ and $\Delta_{4}-V 2$ as a function of electric field for relaxed and strained $\mathrm{Si}_{1-x} \mathrm{Ge}_{x}$ with different $x$. As an overall trend, the generation rate is increasing with $x$ at a given electric field. This is mainly due to the reduction of $E_{g}$ with increasing $x$. Strain, however, impacts both bandgap and masses. When $x \leq 0.25$, strain degrades

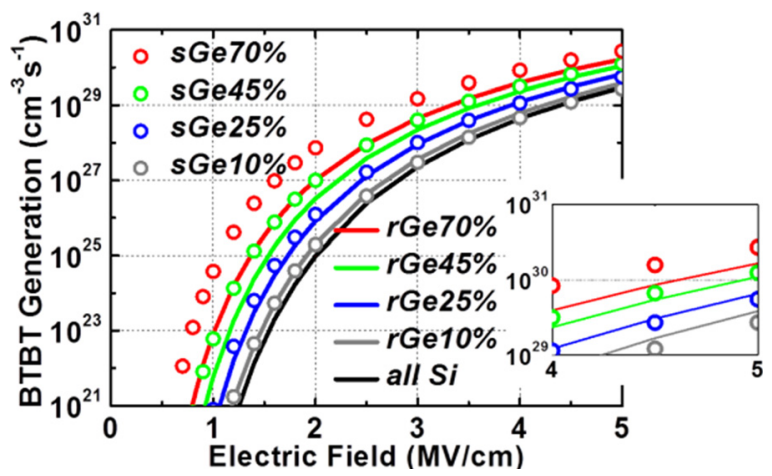

FIG. 11. BTBT generation rate of relaxed and strained $\mathrm{Si}_{1-x} \mathrm{Ge}_{x}$ as a function of uniform electric field. The tunneling direction is along op[001]. The inset presents a zoom-in version showing the degraded generation rate due to strain when $x \leq 0.25$ (the all-Si curve is removed for clarity). the BTBT generation rate at the high electric field (inset in Fig. 11), which is due to the abrupt reduction of $A_{\text {ind }}$ of the $V 1$ band presented in Fig. 10(a). When $x \geq 0.25$, the strained BTBT generation rate exceeds the relaxed one, which is attributed to the dominance of the reduction of $E_{g}$ associated with the $V 1$ band as shown in Fig. 10(b). Note that the $A_{\text {ind }}$ is determined by considering the DOS masses at two band edges. A large $A_{\text {ind }}$ is expected with a wider energy window $(100 \mathrm{meV}){ }^{36}$

\section{BTBT MODEL CALIBRATION WITH SENTAURUSDEVICE}

BTBT model calibration is performed based on a comparison of strained $\mathrm{Si}_{0.75} \mathrm{Ge}_{0.25}$ and strained $\mathrm{Si}_{0.55} \mathrm{Ge}_{0.45}$ diode electrical measurements with SIMS-based diode simulations. First, the SIMS profiles are imported into SProcess defining the doping profiles and material composition. The SProcess simulations provide the resulting strain profiles due to the lattice mismatch between $\mathrm{Si}$ and $\mathrm{SiGe}$. The initial $\mathrm{Si}(001)$-sub is $100 \mu \mathrm{m}$ wide. Only the central part ( $1 \mu \mathrm{m}$ wide) of the substrate, which retains a uniform strain profile, is exported for the SDevice simulations. The validation of this approach is bolstered by the fact that the smallest measured diode is $50 \times 50 \mu \mathrm{m}^{2}$, while the nonuniform strain profile merely extends a few hundreds nanometers from the mesa sidewall according to the simulation results (not shown). In addition, the electrical current is dominated by areal current rather than perimeter current as presented in Fig. 3.

To enhance the accuracy of the calibration, $C V$ measurements are performed as well. Because these diodes are 

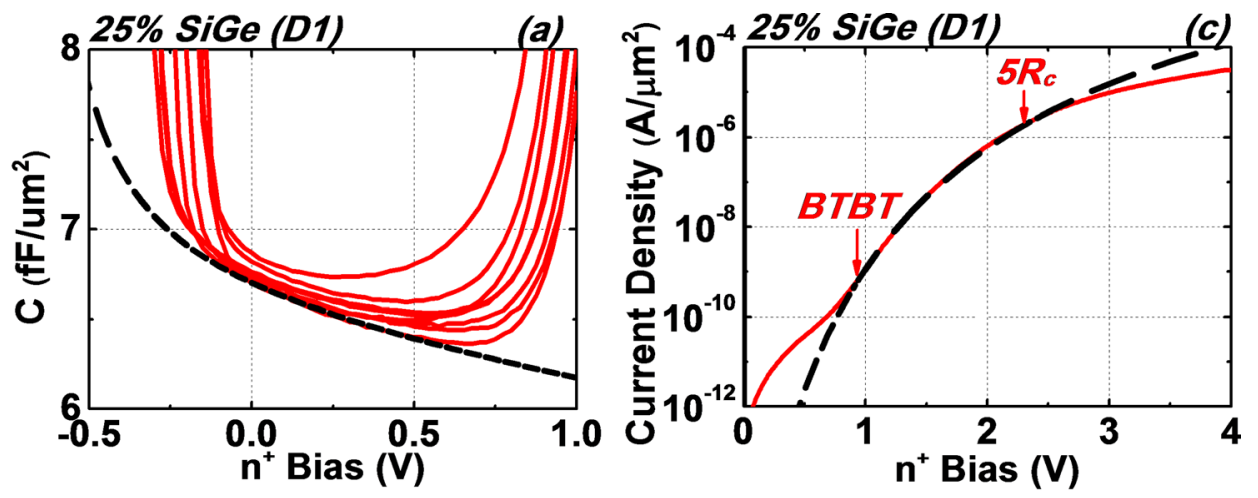

FIG. 12. (a) and (b) Experimental (solid red) and simulated (dashed black) $C V$ curves at $1 \mathrm{MHz}$. Solid $\mathrm{CV}$ lines are measurements of different samples. Simulations deviate from measurements at high bias because trap-related and tunneling models cannot be coupled in CV simulations. $^{25}$ (c) and (d) Experimental (solid) and calibrated simulations (dashed) of the $I V$-curves. The electric field in the intrinsic SiGe region for voltages between the arrows indicating the onset and end of the BTBT region (BTBT and $5 R_{c}$, respectively) ranges from 1 to $1.8 \mathrm{MV} / \mathrm{cm}$ for D1 and from 1 to $1.5 \mathrm{MV} / \mathrm{cm}$ for D2. (Diode dimension: $50 \times 50$ and $70 \times 70 \mu \mathrm{m}^{2}$ for $I V$ and $\mathrm{CV}$ curves, respectively. All figures share the same legend.)
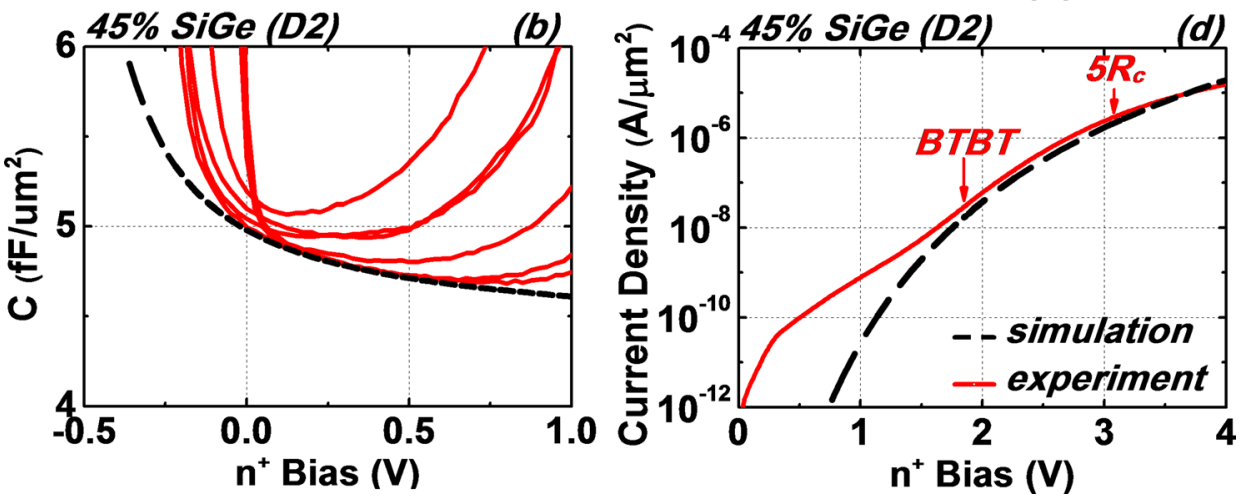

designed for BTBT calibrations, mapping doping profiles or extracting the band offsets at the hetero-interface by $\mathrm{CV}$ measurements are not possible due to the large tunneling leakage currents under high reverse bias. However, the depth accuracy of the SIMS profiles can be improved by making use of the $C V$ characteristics at low bias. Detailed simulation analysis has shown that two dopant profiles resulting in the same $C V$ characteristics at low bias also have the same BTBT characteristics. ${ }^{13}$ Figs. 12(a) and 12(b) show the experimental and simulated (with the wide-most doping profiles) $C V$ characteristics of D1 and D2. As can be seen, good matching is obtained between experiments and simulations with the modified SIMS profiles within the $\pm 0.1 \mathrm{~V}$ voltage window. Note that the central (narrow-most) doping profiles result in about $25 \%(140 \%)$ and $20 \%$ (45\%) larger capacitance values than experiments at zero bias for D1 and D2, respectively. The wide-most profiles will therefore be used for the BTBT calibration. The voltage window is limited to avoid leakage current, namely, thermal diffusion current and tunneling currents in the forward and reverse bias, respectively. The shape of the $C V$ curve is expected, since capacitance values are generally lower with increasing the reverse bias (positive $\mathrm{n}^{+}$bias) due to the longer depletion length.

Limitations of the SDevice model implementation require great care in specifying the SDevice parameter file. The phonon-assisted BTBT model is based on the effective mass approximation in the tunneling direction. SDevice dynamically searches the tunneling paths and correctly determines the tunneling length by including all BGN models aforementioned. SDevice uses $A_{\text {ind }}$ and $B_{\text {ind }}$ internally to determine the parameters needed in the Wentzel Kramer Brillouin (WKB) path integrals, such as masses $\left(m_{c}, m_{v}\right.$, and $\left.m_{r}\right)$, $g, \rho$, and phonon parameters. However, the bandgap in Eqs. (2) and (3), which is used for this parameter extraction, is the relaxed one specified in the parameter file rather than the one including BGN models. ${ }^{25}$ This implies that to get a correct extraction of parameters, the parameter file in SDevice cannot take the values of Table III, rather the formulas in Eqs. (2) and (3) have to be recalculated with the relaxed bandgap. Second, only one value for $A_{\text {ind }}$ and $B_{\text {ind }}$ can be specified for each region, such that in general configurations, non-uniform doping or strain profiles may require a division of the structure in narrow regions, each with its own $A_{\text {ind }}$ and $B_{\text {ind }}$ parameters. In our strained $\mathrm{p}^{+}-\mathrm{i}-\mathrm{n}^{+} \mathrm{SiGe}$ diodes, the Ge, strain profiles are not uniform (Fig. 2). However, with each Ge concentration, only one strain value is associated, such that one set of $A_{\text {ind }}$ and $B_{\text {ind }}$ can be linked to each $\mathrm{Si}_{1-x} \mathrm{Ge}_{x}$ compound. Moreover, concerning the nonuniform doping, all material properties (except for the bandgap) in Eqs. (2) and (3) are independent of doping concentration. Therefore, if $A_{\text {ind }}$ and $B_{\text {ind }}$ in Table III are rescaled by the relaxed bandgaps, correct BTBT simulations result, because this implementation results in the proper values of strained masses, $g, \rho$ and phonon parameters, which are internally used for BTBT generation rate calculations.

Figs. 12(c) and 12(d) show a comparison of experiments and simulations with the calibrated BTBT model. The SiGe diode simulation is implemented including both transitions $\left(\Delta_{4}-V 1\right.$ and $\left.\Delta_{4}-V 2\right)$. The calibration window is indicated by two arrows (BTBT and $5 R_{c}$ ). The BTBT arrow is identified by temperature measurements (Fig. 4). The contact resistance $R_{c}$ is extracted in the high voltage range, and beyond the $5 R_{c}$ arrow, $20 \%$ or more of the total $I V$ resistance including tunneling resistance originates from $R_{c}$.

In the BTBT model calibration, a multiplication of the values of $A_{\text {ind }}$ and $B_{\text {ind }}$ of Table III with 7.6 and 1/1.1, respectively, had to be applied to obtain the excellent fit between simulation and experiment of Figs. 12(c) and 12(d). 
This is consistent with a separate study on relaxed all-Si diodes, which had indicated the same multiplication factors (not shown here). Note that the same multiplication factors are applied for the two different Ge contents shown in Figs. 12(c) and 12(d), as well as for both transitions $\left(\Delta_{4}-V 1\right.$ and $\left.\Delta_{4}-V 2\right)$.

The $10 \%$ decrease in $B_{\text {ind }}$ compared to theory can be attributed to an uncertainty in $m_{r}$, since the bandgaps used in the calculation of Eq. (3) are obtained experimentally and are expected to be well-known. The $10 \%$ decrease in $B_{\text {ind }}$, if real, would correspond to a $20 \%$ lighter mass than theory predicts. Part of this reduction, however, is expected to be due to the effective mass approximation used in SDevice, which overestimates the attenuation in the forbidden bandgap and hence requires a smaller mass input to compensate for this overestimation. As for $A_{\text {ind }}$, even if it is assumed that either all relevant masses $\left(m_{c}, m_{v}\right.$, and $\left.m_{r}\right)$ or only the reduced mass $\left(m_{r}\right)$ are $20 \%$ smaller than theory, while retaining the values of $g, \rho$, and $E_{g}$ as well as the values related to the electron phonon coupling, it is found from Eq. (2) that the ratio of the calibrated $A_{\text {ind }}$ to the theoretical $A_{\text {ind }}$ should be about 10 or 6 , respectively.

The model for $A_{\text {ind }}$ (Eq. (2)), in particular, the factor $\left(m_{D O S, C} m_{D O S, V}\right)^{3 / 2}$, is based on the effective mass approximation. Manku and $\mathrm{Fu}^{35,36}$ indicate that the LH-valence band is indeed rather parabolic up to $100 \mathrm{meV}$ from the band edge, but the HH-valence band is non-parabolic with increasing DOS mass as the energetic distance to the band edge increases. Tunneling beyond $100 \mathrm{meV}$ from the band edge is not expected to contribute significantly, as the exponential factor in Eq. (1) then decreases with at least a factor of 20 due to the corresponding increase in effective bandgap $E_{\mathrm{g}}$. The value of $m_{D O S, V 1}$ at an intermediate energy of $50 \mathrm{meV}$ beyond the band edge is larger with a factor of 2 $\left(\mathrm{Si}_{0.75} \mathrm{Ge}_{0.25}\right)$ to $3\left(\mathrm{Si}_{0.55} \mathrm{Ge}_{0.45}\right)$. Such an increase would result in an increase of $A_{\text {ind }}$ with a factor of 3 to 5 , which can be seen as an upper limit for the discrepancy between the presented theory and experiment, so not explaining the full difference. Our calibration experiments, therefore, indicate that also the electron-phonon coupling has been underestimated. In particular, the deformation potential is a parameter which is difficult to predict theoretically and only for the TA deformation potential, a predictive formula exists. As aforementioned, the TA phonon is the only phonon considered in the BTBT modeling. This study, therefore, shows that most likely other types of phonons participate in the phononassisted BTBT, such as transverse optical (TO) phonons, ${ }^{38}$ explaining the underestimation of the theoretical $A_{\text {ind }}$. If the factor of 6 to 10 is exclusively attributed to TO phonons, then this would result in deformation potential values of $10 \times 10^{10}$ to $13 \times 10^{10} \mathrm{eV} / \mathrm{m}$.

In Fig. 13, the BTBT generation profiles compared to the Ge content and band edge diagram are shown. As can be seen, the electron and hole BTBT generation occur over the entire intrinsic region with a peak in the respective rates near the $\mathrm{Si}_{x} \mathrm{Ge}_{1-x}$-Si hetero-interface. The latter is related to the smaller dielectric constant of the Si. This peak in generation corresponds to a transition from $E_{v}$ of $\mathrm{SiGe}$ to $E_{c}$ of Si. Since the electron masses of relaxed $\mathrm{Si}$ and compressively strained SiGe are the same (see Table III), and since there is no conduction band offset (see Sec. II), the tunneling probability is not significantly affected by the heterojunction transition, rather mainly dependent on electric field and path length. Therefore, the calibrated model can quantitatively predict the BTBT currents through strained all-SiGe diodes, albeit the BTBT generation profiles do not completely fall in the uniform i-SiGe sections. The calibrated $A_{\text {ind }}$ and $B_{\text {ind }}$ can be found in Table III, which assumes that the scaling factors can be applied to other Ge content also.

\section{PREDICTIONS FOR BIAXIALLY STRAINED SIGe TFETS}

The most promising configuration which is free of size-confinement, a pocketed vertical-tunneling TFET configuration $^{42,43}$ (Fig. 14), is used to evaluate the maximum performance of a TFET based on compressively strained SiGe whereby the tunneling is in the out-of-plane
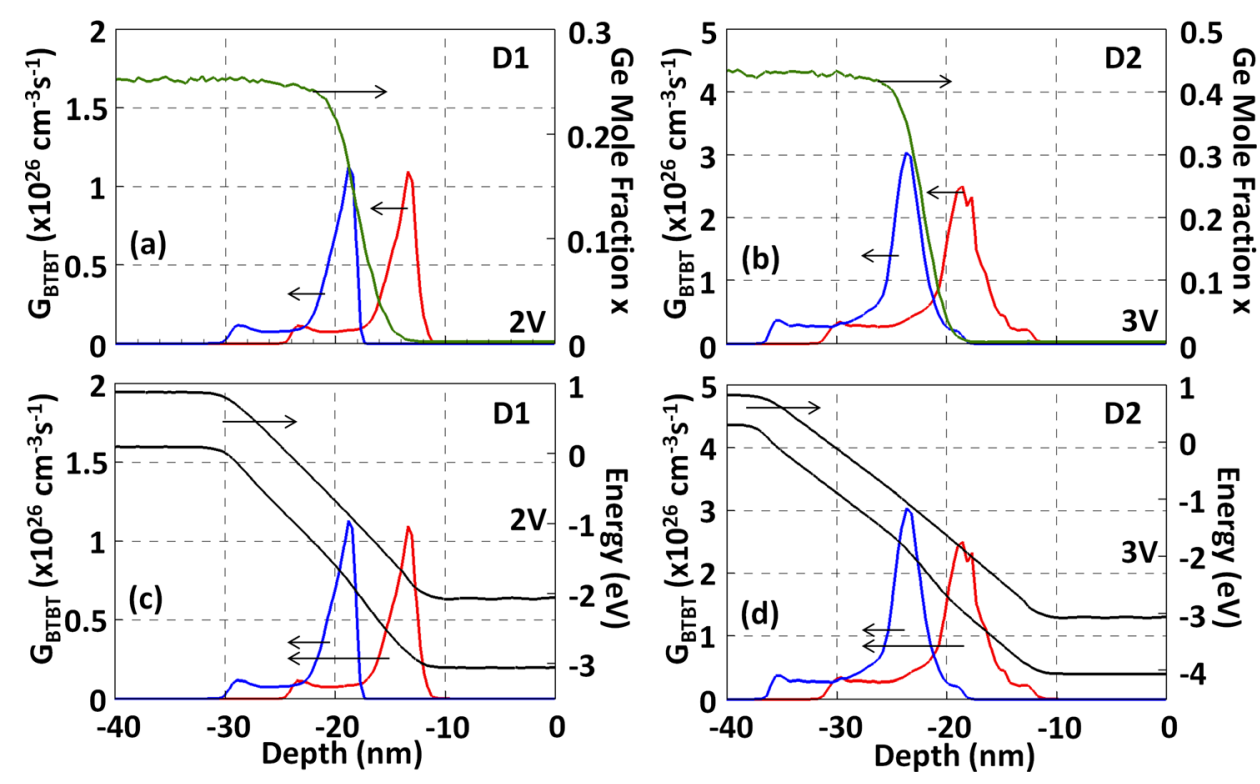

FIG. 13. BTBT generation rates (blue (left) and red (right) lines are for holes and electrons, respectively) compared to Ge mole fraction (a) and (b), and energy band profiles (c) and (d) over the diode cross section of (a) and (c) D1, and (b) and (d) D2 at reverse bias of 2 and $3 \mathrm{~V}$, respectively. The region with high generation rate expands into the intrinsic SiGe section from $\mathrm{Si}$ with increasing the reverse bias. It is the result of the staggered band alignment and the smaller permittivity of $\mathrm{Si}$. 

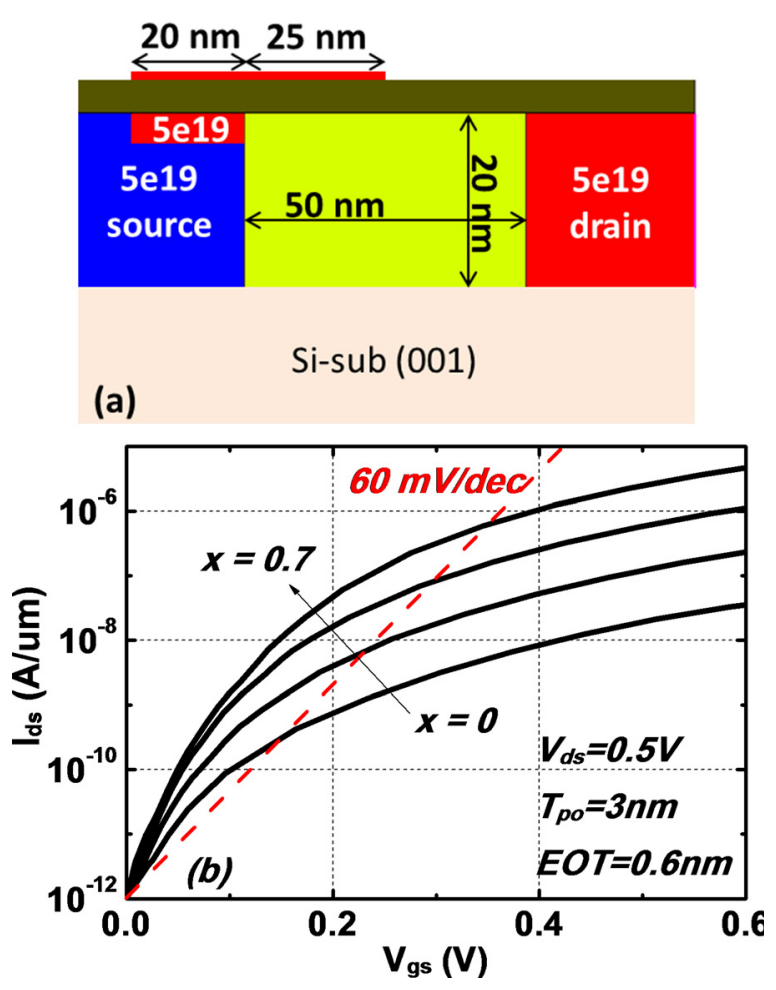

FIG. 14. (a) A pocketed vertical tunneling device. (b) Input characteristics of strained $\mathrm{Si}_{1-x} \mathrm{Ge}_{x}$ TFETs of (a) with $x=0,0.25,0.45$, and 0.7. All curves are shifted to $V_{g s}=0 \mathrm{~V}$ at $I_{d s}=1 \times 10^{-12} \mathrm{~A} / \mu \mathrm{m}$ for ease of comparison. Note that simulation results pose the upper bound of device performance without considering trap-assisted tunneling, which normally increases the off current and degrades the SS.

direction. The counter-doped pocket is heavily doped and $3 \mathrm{~nm}$ thick, which is expected to be able to mitigate the field-induced quantum confinement (FIQC) and the lateral tunneling component. ${ }^{44}$ For the best performance, the gatechannel overlap is introduced to mitigate the series resistance. ${ }^{44}$ Note that the active region is a fully strained SiGe layer above a $\mathrm{Si}$-sub, such that there is homojunction tunneling. To show the upper bound of the device performance, the strain profile is assumed to be uniform throughout the active region, implying that the mesa edge relaxation is ignored, and abrupt doping profiles are assumed in the simulations.

All physical models used in simulations are the same as those aforementioned for correct Fermi level positioning and bandgap reduction due to strain and doping concentration. Simulations are carried out by using the calibrated $A_{\text {ind }}$ and $B_{\text {ind }}$, and the results are presented in Fig. 14. Though the performance of this device is improved with increasing $x$, the $I_{o n}$ can only reach $2.5 \mu \mathrm{A} / \mu \mathrm{m}$ at $V_{D D}=0.5 \mathrm{~V}$ for $x=0.7$. This is too small to be practically useful. ${ }^{45}$ Moreover, since the large lattice mismatch (up to $4.2 \%$ between $\mathrm{Ge}$ and $\mathrm{Si}$ ) constrains the strained layer thickness, it is very difficult to fabricate the device with very high $x$, hence the highest $x$ is limited to 0.7. Even then, the critical thickness ${ }^{46}$ of $\mathrm{Si}_{0.3} \mathrm{Ge}_{0.7}$ grown on $\mathrm{Si}(001)$-sub is about $3 \mathrm{~nm}$. So it would already be very challenging to make the defect-free structure of Fig. 14(a) since the pocket alone is taken to be $3 \mathrm{~nm}$ thick.

\section{CONCLUSION}

Kane's BTBT model has been calculated for biaxially and compressively strained SiGe by incorporating the experimental data of bandgaps, modeled effective masses, and phonon properties. This model is then calibrated by SiGe p-i-n diode measurements and results in scaling factors of 7.6 and 1/1.1 of the parameters $A_{\text {ind }}$ and $B_{\text {ind }}$, respectively. These scaling factors are expected to be due to a limited uncertainty in the relevant effective masses for BTBT, the effective mass approximation, and the absence of TO phonon contributions in the original model. The calibration hence allows an upper limit of the TO-deformation potential value.

Based on the calibrated indirect BTBT model, a prediction for the compressively strained SiGe TFETs with BTBT in the out-of-plane direction has been made, which shows unsatisfactory improvement in the $I_{o n}$ in spite of very high Ge mole fractions $\left(\mathrm{Si}_{0.3} \mathrm{Ge}_{0.7}\right)$. It is therefore concluded that the out-of-plane indirect BTBT in compressively strained homogeneous SiGe is inherently inefficient to achieve high $I_{o n}$ for logic applications. Boosters for the device performance are size confined TFET architecture ${ }^{47}$ or enforcing enhanced direct BTBT in these group IV materials by tensile strain. $^{48}$

\section{ACKNOWLEDGMENTS}

This work was supported by imec's Industrial Affiliation Program.

${ }^{1}$ R. Yu, A. Anisha, N. Jin, S.-Y. Chung, P. R. Berger, T. J. Gramila, and P. E. Thompson, J. Appl. Phys. 106, 034501 (2009).

${ }^{2}$ J.-Y. Li, J. C. Sturm, A. Majumdar, I. Launer, and S. Koester, in Device Research Conference (2009), pp. 99-100.

${ }^{3}$ J.-Y. Li and J. C. Sturm, IEEE Trans. Electron Devices 60, 2479 (2013).

${ }^{4}$ E.-H. Toh, G. H. Wang, L. Chan, G. Samudra, and Y.-C. Yeo, Appl. Phys. Lett. 91, 243505 (2007).

${ }^{5}$ O. M. Nayfeh, C. N. Chleirigh, J. Hennessy, L. Gomez, J. L. Hoyt, and D. A. Antoniadis, IEEE Electron Device Lett. 29, 1074 (2008).

${ }^{6}$ Q. T. Zhao, J. M. Hartmann, and S. Mantl, IEEE Electron Device Lett. 32, 1480 (2011).

${ }^{7}$ A. Villalon, C. Le Royer, M. Casse, D. Cooper, B. Previtali, C. Tabone, J. M. Hartmann, P. Perreau, P. Rivallin, J. F. Danlencourt, F. Allain, F. Andrieu, O. Weber, O. Faynot, and T. Poiroux, in Symposium on VLSI Technology (2012), p. 49.

${ }^{8}$ S. M. Sze, Physics of Semiconductor Devices, 2nd ed. (Wiley, New York, 1981).

${ }^{9}$ V. Nathan and N. C. Das, IEEE Trans. on Electron Devices 40, 1888 (1993).

${ }^{10}$ E. O. Kane, J. Appl. Phys. 32, 83 (1961).

${ }^{11}$ D. K. Mohata, D. Pawlik, L. Liu, S. Mookerjea, V. Saripalli, S. Rommel, and S. Datta, in Device Research Conference (2010), pp. 103-104.

${ }^{12}$ A. C. Ford, C. W. Yeung, S. Chuang, H. S. Kim, E. Plis, S. Krishna, C. Hu, and A. Javey, Appl. Phys. Lett. 98, 113105 (2011).

${ }^{13}$ Q. Smets, D. Verreck, A. S. Verhulst, R. Rooyackers, C. Mercking, M. Van De Put, E. Simoen, W. Vandervorst, N. Collaert, V.-Y. Thean, B. Sorée, G. Groeseneken, and M. M. Heyns, J. Appl. Phys. 115, 184503 (2014).

${ }^{14}$ A. G. Chynoweth, W. L. Feldmann, C. A. Lee, R. A. Logan, and G. L. Peason, Phys. Rev. 118, 425 (1960).

${ }^{15}$ P. N. Butchera, K. F. Hulmea, and J. R. Morgana, Solid-State Electron. 5, 358-360 (1962).

${ }^{16}$ W. N. Carr, J. Appl. Phys. 34, 2467 (1963).

${ }^{17}$ M. S. Tyagi, J. Appl. Phys. 12, 106 (1973).

${ }^{18}$ G. A. M. Hurkx, D. B. M. Klaassen, and M. P. G. Kunvers, IEEE Trans. Electron Devices 39, 331 (1992).

${ }^{19}$ P. M. Solomon, J. Jopling, D. J. Frank, C. D’Emic, O. Dokumaci, P. Ronsheim, and W. E. Haensch, J. Appl. Phys. 95, 5800 (2004). 
${ }^{20} \mathrm{H}$. Fritzsche and J. J. Tiemann, Phys. Rev. 130, 617 (1963).

${ }^{21}$ K. H. Kao, A. S. Verhulst, R. Rooyackers, A. Hikavyy, R. Loo, A. Milenin, J. Tolle, H. Dekkers, E. Simoen, V. Machkaoutsan, J. Maes, K. De Meyer, N. Collaert, M. Heyns, C. Huyghebaert, and A. Thean, ECS Trans. 50(9), 965-970 (2013).

${ }^{22}$ J. McCarthy, S. Bhattacharya, T. S. Perova, R. A. Moore, F. Meyer, H. Gamble, and B. M. Armstrong, Scanning 26(5), 235 (2004).

${ }^{23}$ V. Machkaoutsan, ECS Trans. 50(9), 339 (2013).

${ }^{24}$ M. Bauer, Thin Solid Films 520, 3139 (2012).

${ }^{25}$ Sentaurus, Synopsys, Version G-2012.06, 2012.

${ }^{26}$ A. Vandooren, D. Leonelli, R. Rooyackers, A. Hikavyy, K. Devriendt, R. Loo, M. Demand, G. Groeseneken, and C. Huyghebaert, in ISTDM (2012), p. 108.

${ }^{27}$ C. G. Van de Walle, Phys. Rev. B 34, 5621 (1986).

${ }^{28}$ R. Braunstein, A. R. Moore, and F. Herman, Phys. Rev. 109, 695 (1958).

${ }^{29}$ R. People, IEEE J. Quantum Electron. 22, 1696 (1986).

${ }^{30}$ R. People, Appl. Phys. Lett. 48, 538 (1986).

${ }^{31}$ S. C. Jain and D. J. Roulston, Solid-State Electron. 34, 453 (1991).

${ }^{32}$ M. V. Fischetti and S. E. Laux, J. Appl. Phys. 80, 2234 (1996).

${ }^{33}$ T. Manku and A. Nathan, Phys. Rev. B 43, 12634 (1991).

${ }^{34}$ S. K. Chun and K. L. Wang, IEEE Trans. Electron Devices 39, 2153 (1992).

${ }^{35}$ T. Manku and A. Nathan, J. Appl. Phys. 69, 8414 (1991).

${ }^{36}$ Y. Fu, S. C. Jain, M. Willander, and J. J. Loferski, J. Appl. Phys. 74, 402 (1993).

${ }^{37}$ C. Rivas, Ph.D. dissertation, Department of Electrical Engineering, University of Texas, Dallas, TX, 2003.
${ }^{38}$ R. A. Logan, J. M. Rowell, and F. A. Trumbore, Phys. Rev. 136, A1751 (1964).

${ }^{39}$ A. G. Chynoweth, R. A. Logan, and D. E. Thomas, Phys. Rev. 125, 877 (1962).

${ }^{40}$ M. E. Levinshtein, S. L. Rumyantsev, and M. S. Shur, Properties of Advanced Semiconductor Materials: GaN, AlN, InN, BN, SiC, SiGe (Wiley, New York, 2001).

${ }^{41}$ K. H. Kao, A. S. Verhulst, W. G. Vandenberghe, B. Sorée, G. Groeseneken, and K. De Meyer, IEEE Trans. Electron Devices 59, 292 (2012).

${ }^{42}$ K. H. Kao, A. S. Verhulst, W. G. Vandenberghe, and K. De Meyer, IEEE Trans. Electron Devices 60, 6 (2013).

${ }^{43}$ D. Verreck, A. S. Verhulst, K. H. Kao, W. G. Vandenberghe, K. De Meyer, and G. Groeseneken, IEEE Trans. Electron Devices 60, 2128 (2013).

${ }^{44}$ K. H. Kao, A. S. Verhulst, W. G. Vandenberghe, B. Soree, W. Magnus, D. Leonelli, G. Groeseneken, and K. De Meyer, IEEE Trans. Electron Devices 59, 2070 (2012).

${ }^{45}$ International Technology Roadmap for Semiconductors, 2012.

${ }^{46}$ J. C. Bean et al., J. Vac. Sci. Technol. A 2, 436 (1984).

${ }^{47}$ A. Villalon, C. Le Royer, P. Nguyen, S. Barraud, F. Glowacki, A. Revelant, L. Selmi, S. Cristoloveanu, L. Tosti, C. Vizioz, J.-M. Hartmann, N. Bernier, B. Prévitali, C. Tabone, F. Allain, S. Martinie, O. Rozeau, and M. Vinet, in Symposium on VLSI Technology (2014), p. 1.

${ }^{48}$ K. H. Kao, A. S. Verhulst, M. Van de Put, W. G. Vandenberghe, B. Soree, W. Magnus, and K. De Meyer, J. Appl. Phys. 115, 044505 (2014). 